Recepción: 08/ 06/ 2016

Aceptación: 29 / 09/ 2016

Publicación: 15/ 11/2016

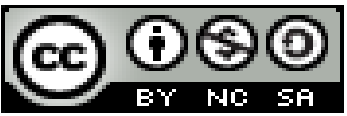

Ciencias técnicas y aplicadas

Artículo de investigación

\title{
Aplicación de la tecnología termo-acústica-cavitacional (TAC) para el procesamiento de residuos de hidrocarburos
}

Application of thermo-acoustic-cavitational technology (TAC) for the processing of hydrocarbon residues

\section{Aplicando a tecnologia de termo-acústico-cavitational (TAC) para o processamento de resíduos de hidrocarbonetos}

Iván Vladimir Nieto-Guerrero I

ivan.nieto@eppetroecuador.ec

Edwin Dimitri Nieto-Guerrero II

ednieto@uce.edu.ec

Edward Jiménez III

IIIEdwardjm@gmail.com

Correspondencia: ivan.nieto@eppetroecuador.ec

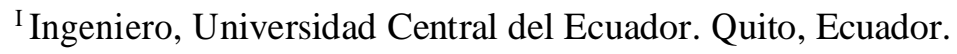

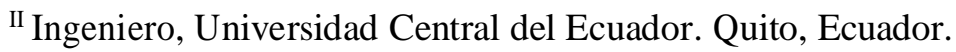

III Ingeniero, Universidad Central del Ecuador. Quito, Ecuador. 


\section{Resumen}

En el proceso de modernización de nuestras refinerías, incluida la Refinería Estatal Esmeraldas (REE), se tiene que propender a aumentar su eficiencia; es decir, a incrementar sustancialmente la conversión de las mismas, a través de la utilización del residuo como materia prima para la obtención de productos livianos.

A fin de incrementar la conversión, consecuentemente la eficiencia de nuestras refinerías en un cercano futuro, una de vías plantea el presente artículo. Para iniciar, propone replicar un proyecto de aplicación de la tecnología termo-acústica-cavitacional primero en un laboratorio de una universidad y, dependiendo de los resultados, en una planta piloto de 100 bls/día de capacidad para procesamiento de residuos de hidrocarburos.

A diferencia de los esquemas clásicos, la nueva Tecnología Termo-Acústica-Cavitacional permite incrementar la profundidad de craqueo térmico de los hidrocarburos y descarta la formación de coque. El residuo de petróleo se somete a un craqueo suave con termólisis cavitacional y acústica posterior. El residuo de termólisis, en condiciones del proceso, se convierte en una fracción de gasolina-diésel y fracción C3-C4, alcanzando un rendimiento ligeramente superior al $50 \%$ volumétrico de fracciones livianas, por un lado. Por otro lado, se obtiene residuos secundarios o asfalto de alta calidad.

En un centro de refinación para producir combustibles de alta calidad, EURO 4 o 5, la tecnología Termo-Acústica-Cavitacional (TAC) permite excluir las unidades de destilación al vacio, craqueo catalítico y coquificación dentro del esquema de procesamiento. Sin embargo, siendo un proceso base, requerirá de hidrotratamiento de destillados; planta de hidrogeno; bloque de producción de azufre; de asfalto; de isomerización y reformado catalítico.

La evaluación económica preliminar para el procesamiento de 10.000 bls/dia del residuo de Shushufindi con la aplicación de la tecnología TAC, tomando en consideración los precios de importación y exportación de derivados desde el 2005 hasta el noviembre 2015, reportados en los informes estadísticos de EP PETROECUADOR 2005-nov-2016, y ajustados por transporte hasta Shushufindi, arroja resultados satisfactorios: una TIR promedio de $19.73 \%$, un VAN promedio de 157,46 millones de dólares y una rentabilidad promedio de 1,63 dólares por cada dólar invertido. 
Palabras clave: modelo económico; equilibrio económico; tasa interna de retorno; contrato; plan de desarrollo; unidad estructural compleja; tecnología termo-acústica-cavitacional.

\section{Abstract}

In the process of modernization of our refineries, including Esmeraldas (REE) State Refinery, must be tending to increase efficiency; that is, to substantially increase the conversion of the refineries, through the use of the residue of oil for the production of light products.

In order to increase the conversion, consequently the efficiency of our refineries in the near future, one way raises this article. To start with, this paper proposes a project Implementation Of ThermoAcoustic-Cavitational technology in a laboratory of a university and then, depending on the results, in a pilot plant of $100 \mathrm{bbl} /$ day capacity for processing oil residue.

Unlike the classical schemes, the new technology rules out the formation of coke and provides for the necessary depth of hydrocarbons destruction. The residue of oil undergoes a mild cracking with subsequent cavitational-and acoustic thermolysis. The residue of the thermolysis, under conditions of the process, is developed to obtain a $\mathrm{C} 3-\mathrm{C} 4$ fraction, gasoline-and-diesel fraction, reaching a yield slightly higher than $50 \%$ volumetric of light fractions, on one hand. On the other hand, it obtains secondary residual or high quality asphalt.

In a center for refining to produce high-quality fuels, EURO 4 or 5, the Thermo-AcousticCavitation Technology (TAC) allows excluding vacuum distillation unit, catalytic cracking and coking within the processing scheme. However, being a base process will require Hydrotreating; Hydrogen; sulfur; asphalt; isomerization and reformate plants.

The preliminary economic assessment, through a cash flow model for processing 10,000 bbl barrels per day of a Shushufindi oil residue with the application of TAC technology, taking into consideration the prices of import and export of hydrocarbon products from 2005 to nov-2016, reported in Petroecuador's statistical reports 2005-nov 2016, and adjusted for transport to Shushufindi, offerings satisfactory overage results: IRR of $19.73 \%$, a net present value (NPV) of 157.46 million dollars and a return of $\$ 1.63$ for every dollar invested.

Key words: economic model; economic balance; internal rate of return; contract; development plan; complex structural unit; thermo-acoustic-cavitational technology. 


\section{Resumo}

No processo de modernização das nossas refinarias, incluindo Estado Esmeraldas refinaria (REE) deve ser tendendo a aumentar a eficiência; ou seja, para aumentar substancialmente a conversão da mesma, através da utilização de resíduos como matéria-prima para a produção de produtos leves. Para aumentar a conversão, consequentemente, a eficiência de nossas refinarias no futuro próximo, de uma forma levanta este artigo. Para começar, propõe para replicar um projecto aplicação cavitational termo-acústico-primeira tecnologia num laboratório de uma universidade e, dependendo dos resultados, uma capacidade de piloto 100 barris / dia para o processamento da planta resíduo óleo.

Ao contrário dos sistemas clássicos, a nova tecnologia termo-acústicas de cavitação pode aumentar a profundidade de craqueamento térmico de hidrocarbonetos e de formação de coque descartados. O resíduo de óleo é submetida a hidrocracking suave e subsequente termólise acústica por cavitação. A termólise resíduo sob as condições do processo, torna-se uma fracção de gasolina e diesel fracção-C3-C4, atingindo um pouco mais de 50\% em volume de fracções leves rendimento de um lado. Por outro lado, é obtido resíduos secundários ou asfalto de qualidade.

Um centro de refinação para a produção de combustíveis de alta qualidade, EURO 4 ou 5, cavilhação termo-acústicas de tecnologia (TAC) permite excluir unidades de destilação de vácuo, o craqueamento catalítico e de coque dentro do esquema de processamento. No entanto, sendo uma base processo, requerem destillados hidrotratamento; planta de hidrogénio; bloco de enxofre de produção; asfalto; isomerização e reformação catalítica.

A avaliação econômica preliminar para o processamento de 10.000 barris / dia de resíduos Shushufindi com a aplicação da tecnologia CT, tendo em preços de importação consideração e exportação de derivados, de 2005 até novembro de 2015, de relatórios estatísticos EP PETROECUADOR 2005 novembro 2016, ajustado para o transporte para Shushufindi, produz resultados satisfatórios: TIR média de 19,73\%, uma média de 157,46 milhões NPV e um retorno médio de US \$ 1,63 para cada dólar investido.

Palavras chave: modelo econômico; equilíbrio econômico; taxa interna de retorno; contrato; plano de desenvolvimento; unidade estrutural complexo; tecnologia termo-acústico-cavitação. 


\section{Introducción}

La capacidad instalada actual de refinación en el país es de 189,500 bls/día: 110,000 en Refinería Esmeraldas; 45,500 en Refinería La Libertad; 20,000 en Refinería Amazonas; 1,000 en Refinería Lago Agrio; 1600 en el Bloque 15; todas estatales; y 11,400 en manos de compañías extractoras de crudo.

El nivel de producción nacional de hidrocarburos livianos: gas doméstico, gasolinas y diésel es bastante bajo y refleja la inadecuada e insuficiente infraestructura de refinación existente en el país, que nos obliga, para satisfacer la demanda interna en calidad y cantidad, a importar grandes volúmenes de combustibles refinados. El único centro de refinación que cuenta con procesos tradicionales de mediana conversión es la refinería Estatal de Esmeraldas, que alcanza un rendimiento de productos refinados cercano al $58 \%$ [3]. Todos los demás son apenas procesos de destilación primaria; es decir procesos de separación física de los hidrocarburos que constituyen el petróleo. En el caso de las refinerías de Shushufindi y de la Libertad, el rendimiento de productos refinados base esta alrededor del $49 \%$ vol. y el residuo está cerca del $51 \%$ vol. [1; Industrialización].

Esta infraestructura de refinación no permite cubrir la demanda interna de combustibles; ya que produce una elevada cantidad de residuos que, luego de mezclarlos con cutter stock (diésel negro de menor calidad) importado, son exportados en calidad de fuel oíl No 4 y/o fuel oíl No 6 [1]. Además, la calidad de los combustibles que se produce en el país dista mucho de sus similares a nivel internacional. Las diferencias están dadas por el bajo número de octano en las gasolinas y alto contenido de azufre en todos los combustibles.

Mientras no se modernicen las actuales refinerías y se construya una nueva, el país continuará importando cada vez mayores volúmenes de gas doméstico (LPG), gasolina de alto octanaje y diésel Premium. En 2005, las importaciones fueron de 24,6 millones de barriles a un costo de US \$ 1627,2 millones de dólares. En el 2015, las importaciones sobrepasaron los 56,3 millones de barriles a un costo de 3.906 millones de dólares. Por otro lado, en 2015, el país exportó 5,7 millones de barriles de Fuel Oíl No 4 y 6 con un ingreso total de 261,1 millones de US dólares. 
En resumidas cuentas, exportamos residuos semielaborados, producidos en las refinerías e importamos productos blancos (LPG, naftas de alto octanaje, diésel 2 y diésel Premium)-

Una de las tareas primordiales de los centros de refinación es mejorar la tecnología de procesamiento del petróleo y sus derivados, a fin de incrementar el volumen de los productos destilados del petróleo. En las refinerías, para aumentar la cantidad de destilados livianos se utilizan principalmente los procesos de craqueo térmico, craqueo catalítico, hidrocraqueo y coquificación.

Las tecnologías básicas para el procesamiento de petróleo y derivados del petróleo son la destilación y el craqueo. Afectar a la cinética de estos procesos se puede a través de productos químicos (catalizadores, sustancias tensioactivas, aditivos, etc.) y mediante métodos físicos (térmicos, cavitacionales, electromagnéticos, etc.) Como resultado de esta intervención varía el radio del núcleo y el espesor de la unidad estructural compleja, que forma parte del sistema disperso del petróleo.

La utilización de productos químicos resulta en un aumento significativo del costo del producto final, en un desgaste acelerado de las columnas de rectificación y es un proceso de difícil regulación. Por esta razón, muchos investigadores han dado una atención especial a los métodos de procesamiento intensivo (físicos) de petróleo y residuos para aumentar el rendimiento de fracciones ligeras y reducir el contenido de azufre.

La tecnología de separación intensiva de hidrocarburos se basa en los efectos termo mecánicos sobre el residuo que se inicia a temperaturas un poco más bajas que el de su craqueo térmico; presencia de cavitación y de vibraciones ultrasónicas, sin el uso de catalizadores. En el aparato para la separación intensiva de hidrocarburos, se acumulan las vibraciones resonantes, que conduce a la ruptura de las uniones en las moléculas, a su craqueo y, en consecuencia, aumenta la producción de derivados livianos.

La aplicación de efectos cavitacionales-acústicos, permite suprimir los procesos de generación de coke e intensifica los procesos de craqueo a causa de la acción micro hidrodinámica de los medios líquidos reaccionantes. 


\section{Materiales y métodos}

En el proceso de modernización de nuestras refinerías se tiene que propender a aumentar su eficiencia; es decir, a incrementar sustancialmente la conversión de las mismas, a través de la utilización del residuo como materia prima para la obtención de productos livianos.

Últimamente han aparecido innovaciones tecnológicas que permiten procesar los residuos y crudos pesados, a través de tecnologías termo-acústicas que requieren de menores temperaturas, bajas presiones y no necesitan de catalizadores sofisticados. Todo esto hace que las inversiones sean sustancialmente menores, aproximadamente un 15-25\% menos que el costo de las tecnologías tradicionales, así como reducen, sustancialmente, un 20-25\% el tiempo necesario para la puesta en marcha de las plantas.

Estos procesos (termo-acústicos) se sustentan en innovaciones tecnológicas realizadas al proceso de craqueo térmico y sobre su base desarrollaron una tecnología innovadora, que permite adicionalmente producir 40-55\% más naftas, fracción diésel y corte para GLP, por un lado. Además, permite producir asfaltos terminados de alta calidad, aprovechando como materia prima el residuo de refinación de crudo.

Con el soporte de un modelo económico de flujo de caja, se analiza el impacto que tendría esta tecnología en el caso de un proyecto industrial para el procesamiento del residuo de Shushufindi, tomando como referencia los resultados de laboratorio, obtenidos al procesar residuo de Shushufindi en el Centro de Investigación de la Cía. Termakat en la ciudad de Ufa-Rusia.

\section{Breve análisis técnico de la tecnología TAC}

En esencia el proceso termo-acústico-cavitacional se explica como la ruptura de la continuidad del medio líquido, debido a un cambio violento, brusco en la velocidad de flujo hidrodinámico, que da como resultado la formación de micro burbujas, que prácticamente en forma instantánea colapsan. A este colapso se llama cavitación, en Inglés "Bubble collapse". Con el colapso (implosión) de la burbuja se libera una energía muy grande. Por ejemplo, el investigador norteamericano Kenneth Hamster en el artículo "los efectos químicos de las ondas sonoras", publicado en la revista »Scientific American № 4, 1989, indica que la temperatura, en el momento del colapso de la burbuja, puede alcanzar $5000{ }^{\circ} \mathrm{C}$, la presión alrededor de 5000 bar, y 
la velocidad de palpitación las paredes de la burbuja alcanzan $450 \mathrm{~km} /$ hora. Hay que precisar que el tamaño efectivo de las burbujas de cavitación es de 5 - 20 micrones y su vida es unas diezmillonésimas de segundo. Según Termakat, la energía que aporta el efecto cavitacional es el resultado del efecto combinado de una gran cantidad de burbujas de cavitación y permite reducir la temperatura del proceso de visbreaking en $30-50{ }^{\circ} \mathrm{C}$. De esta manera se evitan las reacciones en cadena de termopolicondenzación y se evita la formación de coque.

La tecnología TAC rompe la cadena continua de los hidrocarburos, interrumpiendo la comunicación entre las distintas partes de las moléculas, afecta el cambio de la viscosidad estructural, es decir, genera una ruptura temporal de las uniones de Van Der Vaals. Bajo la influencia de la cavitación de alta intensidad durante un lapso de tiempo se interrumpen las uniones C-C en las moléculas de los hidrocarburos, lo que resulta en cambios de la composición físico-química (disminución de peso molecular, la temperatura de cristalización, etc.) y de las propiedades de los derivados (viscosidad, densidad, punto de inflamación, etc.). La aplicación de impulsos cavitacionales-acústicos sobre el residuo libera gran cantidad de energía que se utiliza para romper los enlaces químicos entre los átomos de las grandes moléculas de hidrocarburos compuestos.

La energía de disociación del enlace $\mathrm{C}-\mathrm{H}$, varía según el peso molecular y la estructura de la molécula, está entre 322 y $435 \mathrm{~kJ} / \mathrm{mol}$, la energía de disociación de la unión C-C fluctúa entre 250 y 348 kJ/mol [9]. En la ruptura de las moléculas C-H se separa el hidrógeno, en el proceso de ruptura de las uniones $\mathrm{C}-\mathrm{C}$ la molécula se rompe en dos partes desiguales.

Un impacto energético de los efectos cavitacionales-acústicos sobre el residuo del petróleo permite aumentar la producción en las fracciones más volátiles en el proceso de destilación. La Aplicación del impulso energético, sobre el petróleo pesado, permitiría obtener un 3-5\% de GLP; corte de gasolina: 7-10\%; de combustible diésel: 35-40\% y de residuo secundario, asfalto y otros productos pesados un $45-60 \%$ vol.

\section{Tecnologías existentes}

1. La tecnología Sulphco (http://www.sulphco.com/index.asp) usa el ultrasonido de alta potencia para modificar las estructuras moleculares que ocurren naturalmente en los 
hidrocarburos (petróleo) y el agua. En esencia, las ondas de ultrasonido presionan (estresan) a las burbujas, provocando que crezcan, se contraigan y, eventualmente, se produzca su implosión. Con la implosión, un inmenso calor y presión se producen en y alrededor de cada sub-micrón y micrón de la de burbuja. Todo el ciclo sólo toma unos nanosegundos. Cada implosión de la burbuja actúa como un micro reactor, acelerando las reacciones físicas, debido al inmenso calor liberado a causa de la implosión. Las temperaturas pueden llegar a $10.000{ }^{\circ} \mathrm{K}$, con presiones de hasta 5.000 PSI durante unos nanosegundos al interior de la burbuja implosionante. Estas son las condiciones en las que se rompen los enlaces moleculares.

Las ventajas económicas que presenta esta tecnología, en resumen, son el incremento del precio del crudo y sus derivados por la disminución del contenido de azufre y viscosidad, así como el aumento del grado API y disminución de la viscosidad.

2. La tecnología, conocida como "Visbreaking-TERMAKAT" (http://www.termakat.ru) se sustenta sobre la base del craqueo térmico suave, intensificado por la acción cavitación-acústica en el medio de reacción. En resumen, la energía generada por cavitación y efectos acústicos generan un cambio en la estabilidad hidrodinámica y la dispersión de medios líquidos, que tienen diferentes efectos sobre las diferentes etapas del proceso - en particular la intensificación de unas reaacciones (craqueo, destrucción con generación de hidrocarburos más livianos que la carga) y disminución drástica de otras (poli-condensación con generación de coke).

En el caso de Termakat, como generador de energía cavitacional-acústica se utilizan bombas arregladas de cavitación acústica con control de intensidad de la energía cavitacional. La utilización de esta tecnología reduce de forma significativa la temperatura requerida para el craqueo de las moléculas - hasta $440{ }^{\circ} \mathrm{C}$-, que se encuentra muy por debajo de la zona de coquificación. También se reduce la presión de 2,5 MPa a 0,5-0,2 y disminuye a la mitad el metal requerido para los equipos.

\section{Factibilidad técnica de la nueva tecnología}

Una vez que se repliquen los resultados positivos obtenidos en el laboratorio de la Cia Termakat, que se lo podría realizar en una Universidad, en la siguiente etapa se podrá verificar los rendimientos, pero en una planta piloto semi-industrial. 
Para la planta piloto semi-industrial de conversión profunda de residuo atmosférico con la aplicación de la tecnología "Visco reducción-Termo-cavitacional" se propone desarrollar una versión móvil, modular, adaptable a las refinerías existentes.

La opción semi-industrial se la podría ejecutar junto al CIS; ya que se requeriría un mínimo de capital, debido a que todos los servicios de ingeniería y flujos los provee la planta existente. Esta versión incluiría los siguientes equipos principales:

1. Una bomba de residuo de $\mathrm{Q}=1,0 \mathrm{M} 3 /$ hora, para tomar el residuo del fondo de la columna atmosférica con $\mathrm{t}=350^{\circ} \mathrm{C}$ y entregar al horno con una presión de 1,0 MPa;

2. horno para calentar el residuo hasta $\mathrm{t}=430$ a $450{ }^{\circ} \mathrm{C}$, con quemadores a liquido;

3. Cámara de reacción 1 ra fase (soking cámara): $\mathrm{V}=2,0 \mathrm{M} 3, \mathrm{t}=420$ a $430{ }^{\circ} \mathrm{C}, \mathrm{P}=0,4$ $0,6 \mathrm{MPa}$;

4. Cámara de reacción 2 da fase (soking cámara): $\mathrm{V}=2,0 \mathrm{M} 3 ; \mathrm{t}=390$ a $420^{\circ} \mathrm{C}, \mathrm{P}=0,05$ $0,3 \mathrm{Mpa}$

5. Bomba cavitacional-acústica: $\mathrm{t}=440^{\circ}, \mathrm{Q}=6,3 / \mathrm{h}, \mathrm{P}=0,6-0,8 \mathrm{MPa}$;

6. Intercambiador de calor para enfriar el residuo, $\mathrm{F}=6, \mathrm{M} 3$

El equipo podrá ser colocado en 3 plataformas móviles.

En un esquema simplificado, tanto para una planta de laboratorio o semi-industrial, el flujo resumido del proceso se describe a continuación: La bomba de residuo toma el hidrocarburo del fondo de la columna atmosférica lo eleva la temperatura en el horno y va a la 1ra cámara de reacción. Desde allí, la fase líquida se alimenta a la cámara de reacción de la 2 da etapa. La fase gaseosa de las cámaras de reacción va a través de reguladores de presión y de flujo de nuevo en la zona de alimentación de la columna atmosférica. La fase líquida de la 2da cámara se recicla con ayuda de la bomba de cavitación acústica. El exceso de flujo de residuo secundario se envía a través del intercambiador de calor y de un medidor hacia el flujo residual de la refinería. $\mathrm{Si}$ se decide obtener directamente asfalto de carretera de alta calidad, en vez de residuo secundario, se requeriría, principalmente, de una bomba cavitacional-acústica adicional.

Una posible estructura para refinación de alta conversión con esta tecnología para obtener productos acabados EURO 4, estaría integrada por los procesos tecnológicos siguientes: 
El bloque de rectificación de los productos de termólisis gaseosos;

Hidrotratamiento de destillados;

Planta de hidrogeno;

Bloque producción de azufre;

Bloque producción de asfalto.

Bloque de isomerización y reformado catalítico.

\section{Esquema simplificado de planta piloto}

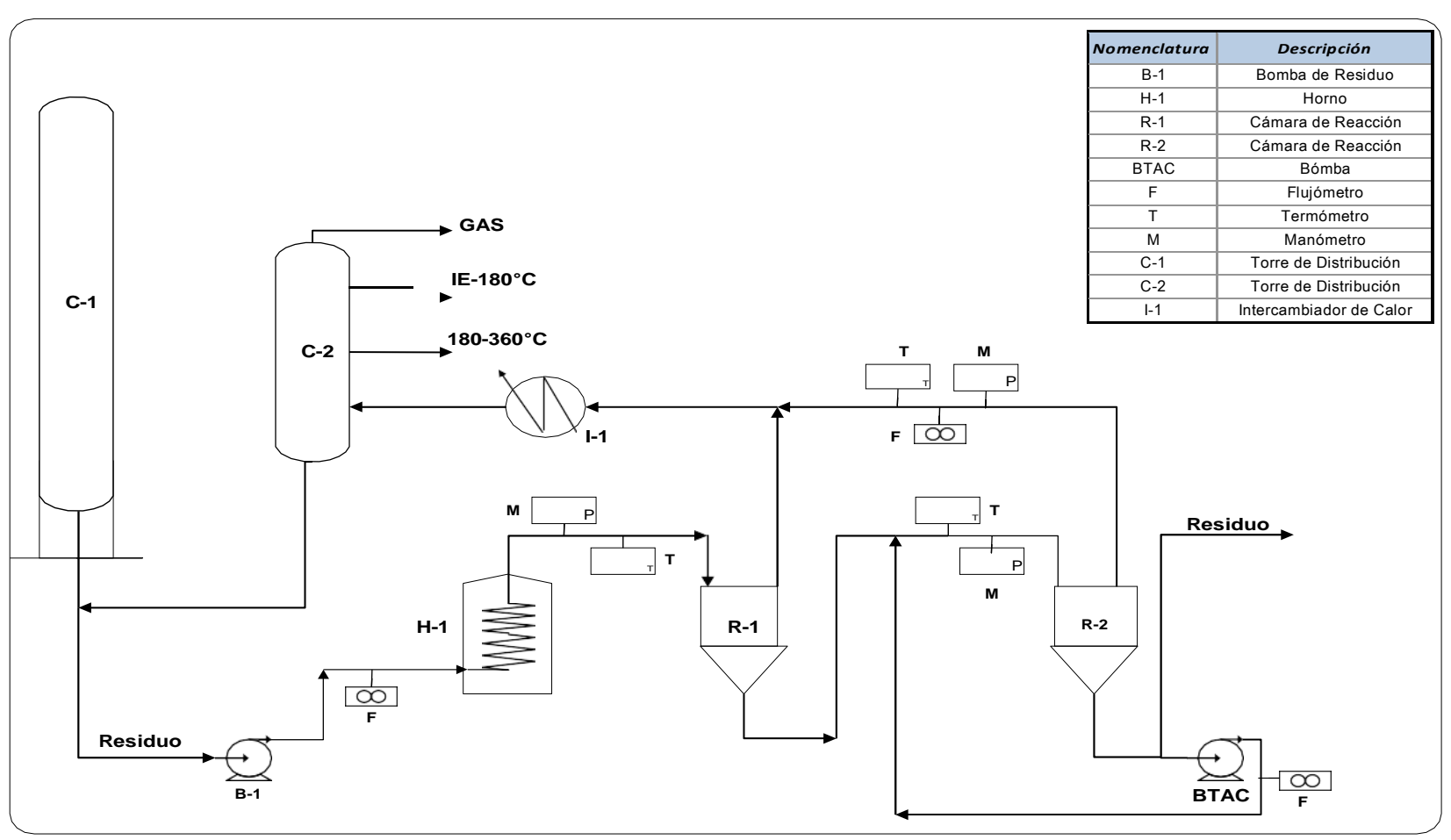




\section{Modelo económico para una planta industrial con tecnología TAC}

Para presentar una evaluación económica preliminar se consideraron como referencia las inversiones calculadas a partir del proyecto que tiene previsto realizar la Filial de Petroindustrial, a fin de modernizar y ampliar la capacidad de procesamiento en la refinería de Shushufindi y se las ajustó solo para el caso de procesamiento del residuo existente. La inversión sería de US \$ 550.000 .000 y se consideró un financiamiento del 50\%. Además, para el presente ejemplo de procesamiento de 10000 bls/d de residuo del CIS, se tomaron en cuenta los rendimientos obtenidos en una de las corridas de la prueba piloto realizadas en el laboratorio de la Cia Termakat y se consideraron los precios $\mathrm{C} \& \mathrm{~F}$ promedio de importación de los combustibles, reportados en los informes estadísticos de PETROECUADOR 2005-2016. Los precios se ajustan por transporte de Esmeraldas a Shushufindi y luego se corren en el modelo de flujo de caja para calcular los índices económicos del proyecto para cada año y para el promedio.

\begin{tabular}{|c|c|c|c|c|c|c|c|c|c|c|c|c|c|}
\hline \multicolumn{14}{|c|}{$\begin{array}{l}\text { PRECIOS DE IMPORTACION MAS FLETE A SHUSHUFINDI (US\$/bl) } \\
\text { E INDICES ECONOMICOS DEL PROYECTO }\end{array}$} \\
\hline $\begin{array}{l}\text { CAPACIDAD PLANTA: } 10000 \\
\text { BLS/DÍA; INVERSIÓN: } 400 \\
\text { MMUS\$ }\end{array}$ & 2005 & 2006 & 2007 & 2008 & 2009 & 2010 & 2011 & 2012 & 2013 & 2014 & 2015 & nov-16 & $\begin{array}{c}\text { PROMEDIO } \\
\text { 2005-2016 }\end{array}$ \\
\hline \begin{tabular}{l|l|} 
& LPG \\
\end{tabular} & 46,8 & 56,7 & 65,4 & 71,7 & 45,3 & 54,92 & 79,17 & 71,44 & 68,67 & 65,28 & 36,16 & 31,56 & 57,8 \\
\hline GASOLINA & 74,7 & 84,8 & 92,19 & 108,78 & 86,03 & 98,98 & 137,69 & 149,58 & 137,40 & 126,08 & 86,38 & 67,5 & 104,2 \\
\hline DIESEL & 79,8 & 82,6 & 89,7 & 123,6 & 82,29 & 96,4 & 136,47 & 140,74 & 136,70 & 124,89 & 74,31 & 57,84 & 102,1 \\
\hline DIESEL PREMIUM & 79,8 & 95,7 & 100,7 & 140,6 & 82,29 & & 139,99 & 142,65 & 138,09 & 126,52 & 85,13 & 65,18 & 108,8 \\
\hline CUTTER STOCK & 61,6 & 73,8 & 71,5 & 110,18 & 91,14 & 90,2 & 122,70 & 127,71 & 121,53 & 113,14 & 61,10 & 48,86 & 91,1 \\
\hline RESIDUO & 27,3 & 31,2 & 49,8 & 62,9 & 47,36 & 61,51 & 84,21 & 89,90 & 88,10 & 74,54 & 21,08 & 23,6 & 55,1 \\
\hline ASFALTO & 39,1 & 52,4 & 51,6 & 57,4 & 57,8 & 57,8 & 57,76 & 57,76 & 57,76 & 57,76 & 57,76 & 57,76 & 55,2 \\
\hline & & & & & & & & & & & & & \\
\hline ORIENTE & 42,84 & 52,80 & 63,68 & 86,96 & 54,34 & 72,57 & 98,92 & 99,49 & 97,36 & 85,81 & 43,44 & 36,24 & 72,0 \\
\hline NAPO & & 48,56 & 61,04 & 79,73 & 50,87 & 69,56 & 95,11 & 96,44 & 92,91 & 81,58 & 39,22 & 30,9 & 67,8 \\
\hline $\begin{array}{r}\text { DIF. DEL PRECIO DE LOS } \\
\text { CRUDOS. }\end{array}$ & & 4,24 & 2,64 & 7,23 & 3,47 & 3,01 & 3,81 & 3,05 & 4,45 & 4,23 & 4,22 & 5,25 & 4,1 \\
\hline $\operatorname{VAN}(\mathrm{AL} 10 \%)$ & 298.615 .972 & 285.976 .783 & 129.183.078 & 243.139 .095 & 108.811 .650 & 35.995 .077 & 67.127 .898 & 24.327 .825 & 13.301.248 & 268.463 .796 & 469.156 .652 & 363.509 .973 & 157.463 .938 \\
\hline TIR $=$ & $22,9 \%$ & $26,6 \%$ & $16,0 \%$ & $20,7 \%$ & $15,1 \%$ & $12,4 \%$ & $13,2 \%$ & $11,2 \%$ & $10,9 \%$ & $14,5 \%$ & $29,1 \%$ & $25,3 \%$ & $19,73 \%$ \\
\hline PAYBACK (AÑOS) & 6,8 & 6,1 & 8,6 & 7,3 & 9,2 & 9,9 & 10,1 & 11,2 & 10,6 & 9,4 & 5,6 & 6,3 & 7,5 \\
\hline $\begin{array}{l}\text { BENEFICIO-COSTO (US\$ NETO } \\
\text { POR } 1 \text { INVERTIDO }\end{array}$ & 0,82 & 1,14 & 0,36 & 0,67 & 0,30 & 0,14 & 0,18 & 0,07 & 0,05 & 0,27 & 1,29 & $1,0 \mathrm{c}$ & 0,63 \\
\hline $\begin{array}{l}\text { INDICE DE RENTABILIDAD } \\
\text { (US\$/US\$.) }\end{array}$ & 1,82 & 2,14 & 1,36 & 1,67 & 1,30 & 1,14 & 1,18 & 1,07 & 1,05 & 1,27 & 2,29 & 2,00 & 1,63 \\
\hline FUENTE DE LOS PRECIOS: INF & MES ES & & & DOR & & & & & & & & & \\
\hline
\end{tabular}




\section{Resultados y Discusión}

Se comprobó plenamente, a nivel de planta piloto de laboratorio, que la tecnología de la cía. Termakat permite obtener altos rendimientos de fracciones livianas (PIE-360 ${ }^{\circ} \mathrm{C}$ ) a partir del residuo de Shushufindi que, en el caso de las pruebas realizadas, superan el $57 \%$ vol. de la carga.

Si esta tecnología se aplicara al residuo de Shushufindi se podría obtener una conversión cercana al $50 \%$ vol., de productos blancos y a partir del crudo una conversión cercana al $75 \%$; muy superior a la existente en la refinería de esmeraldas que apenas alcanza el $58 \%$.

Esta tecnología permite excluir las unidades de destilación al vacio y de coquificación dentro del esquema de procesamiento, razón por la cual generaría ahorros sustanciales en las inversiones, así como en los costos de operación y mantenimiento en la vida del proyecto.

La integración de los procesos termo-acústicos-cavitacionales en el esquema de refinación de Shushufindi permitiría obtener soluciones tecnológicas muy efectivas que permitirían ahorrar centenas de millones de dólares, debido a que requerirían inversiones sustancialmente inferiores, así como tiempos de implementación menores a los estimados en el caso de instalar los procesos de refinación tradicionales.

La evaluación económica preliminar del proyecto analizado para procesar el residuo de Shushufindi, con la tecnología termo-acústica-cavitacional, arroja resultados satisfactorios como una TIR promedio de 19,73\%, un VAN promedio de 157.46 millones de dólares y una rentabilidad promedio de 1,63 dólares por cada dólar invertido.

Si en las plantas piloto de laboratorio y semi-industrial propuestas se ratifican los rendimientos de fracciones livianas obtenidos en laboratorio de termakat con residuo de Shushufindi del CIS, este tipo de tecnología resulta muy conveniente, desde el punto de vista técnico y económico, para incrementar el grado de conversión de nuestras refinerías y reducir sustancialmente las importaciones de combustibles. Proyectos como el propuesto tendrían un gran impacto positivo para nuestro país; ya que, además, impulsaría a nuestra industria metalmecánica que se podría encargar de una gran parte de la fabricación de los equipos y recipientes necesarios para la modernización de las plantas del CIS. También permitiría una alta participación de los técnicos de 
EP PETROECUADOR en las etapas de ingeniería conceptual, básica y de detalle, supervisión de la construcción de los equipos, ensamblaje, interconexión, pruebas en frio y caliente, arranque y funcionamiento; generando así una enorme transferencia de tecnología.

\section{Referencias bibliográficas}

Informes estadísticos de la industria petrolera ecuatoriana 1972-2015. Gerencia de economía y finanzas; planificación corporativa. Petroecuador

Estadístico enero-noviembre 2016. Planificación corporativa. Petroecuador

Presentación sobre situación actual, objetivos, y actividades estratégicas para la industria de refinación ecuatoriana. Ivan Vladimir Nieto Guerrero, dic. 2006. 26 pág

Profundización de la conversión del petroleo. Procesos asequibles. Publicación mercado de combustibles 05 de junio 2010; pág. 22-24. Kuroshkin a.k

Kurochkin Alejandro, Kurochkin av, diseño de pequeñas refinerías con alta conversión; территория нефтегаз (territorio del gas y petróleo); sección refinación de gas y petróleo edición 2; febrero 2010. Moscu. - 18 págs

Incremento de la rentabilidad de pequeñas refinerías por el aumento de producción de la fracción diesel y de asfalto. Revista territorio de petróleo y gas. Marzo 2009 no 3; pág. 33-37. Kuroshkin A.A. y Kuroshkin A.A

Ultrasound craks down to up value. Carrie Ellis. www.chem.info. March 2009.

Complejo industrial Shushufindi. Repotenciación de la refinería amazonas 2007.

Composición del residuo de Shushufindi.

Resultados del informe de comisión de servicios al laboratorio de la Cia A Termakat.

Modelo económico del flujo de caja. 